\title{
Soft Lithographic Fabrication of Microresonators
}

\author{
A. M. Armani and K. J. Vahala \\ Department of Applied Physics, California Institute of Technology \\ 1200 E. California Blvd, M/C 128-95, Pasadena, CA 91125
}

Abstract-Using ultra-high-Q toroid microcavity masters, soft lithography is applied to fabricate polymer microcavity arrays with $Q$ factors in excess of $10^{6}$. This technique produces resonators with material-limited quality factors.

\section{INTRODUCTION}

In typical polymer resonators fabricated using replicating techniques, the dominate loss mechanism is surface scattering which is the result of lithographic imperfections in the initial master structure.[1-3] Therefore, while the material loss of the replicating material (such as PMMA) could achieve quality factors as high as 100 million or greater in the visible, the highest Q's which have been realized in planar polymer devices are $\sim 10,000-40,000$. [2, 3]

In the present work, the initial master used is the ultra-high-Q microtoroid[4]. These silica-based structures exhibit very low surface scattering as evidenced by Q factors in excess of 100 million. As such, they function as an ideal master structure and also allow us to test the replication process. By using this planar device as the master, nearly absorption-limited planar polymer micro-cavity arrays with $\mathrm{Q}$ factors in excess $10^{6}$ can be fabricated. This represents a 40x improvement in Q.[5]

\section{FABRICATION AND TESTING}

The fabrication process flow has 3 steps: fabricate the silica ultra-high-Q toroid master, cast the negative mold from Polydimethylsiloxane (PDMS), and cast the positive mold

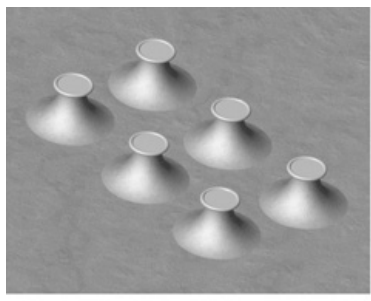

(a)

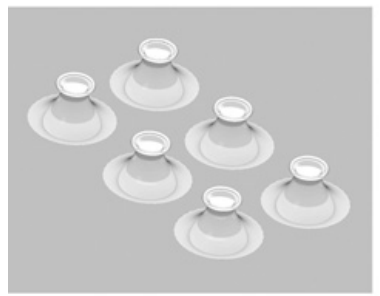

(c)

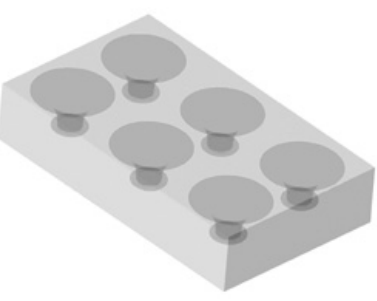

(b)

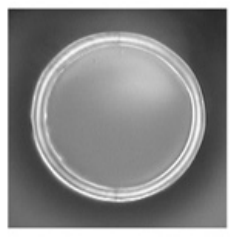

(d)
Fig. 1. The soft lithographic fabrication process. A) an array of ultra-high-Q microtoroids is silanated using TMCS, b) the negative PDMS mold is formed, c) the ngative mold is filled with the polymer of choice, and the polymer reosnators are released, d) an optical micrograph of a Crystal Cast resonator with a diameter of $110 \mu \mathrm{m}$. from the polymer of interest $[5,6]$. The process used to create the ultra high-Q microtoroids is contained in reference 5. The fabrication outline for the polymer microresonators is in figure 1. The ultra-high-Q masters provide an excellent starting point for the soft-lithographic technique and specifically its ability to achieve smooth resonator interfaces.

Polymer resonators were tested using optical fiber taper waveguides.[7, 8] Transmission spectra were taken through tapers coupled to polymer microresonators having diameters between $60-120 \mu \mathrm{m} .[5,6]$ Spectral bands near $680 \mathrm{~nm}, 980 \mathrm{~nm}$, $1300 \mathrm{~nm}$ and $1550 \mathrm{~nm}$ were scanned using single frequency tunable lasers.

\section{RESULTS}

Figure 2 is a typical transmission scan taken near $1550 \mathrm{~nm}$ The observed $\sim 8 \mathrm{~nm}$ free spectral range is consistent with the diameter of the resonator. The full-width at half maximum (FWHM) of the resonances were measured under various taper-coupling conditions to infer the intrinsic Q factor of the devices. In the $1300 \mathrm{~nm}$ band and $1550 \mathrm{~nm}$ intrinsic $\mathrm{Q}$ factors in excess of 100,000 were routinely measured using Vicast, PDMS, Efiron, and Crystal Cast.

To determine the dominate loss mechanism, the loss values of PDMS were determined from values published by the manufacturer and from Metricon measurements. In figure 3, these known loss values are compared with those measured using the microresonator at $980 \mathrm{~nm}, 1300 \mathrm{~nm}$ and $1550 \mathrm{~nm}$. The values were consistent throughout the near-IR spectrum.

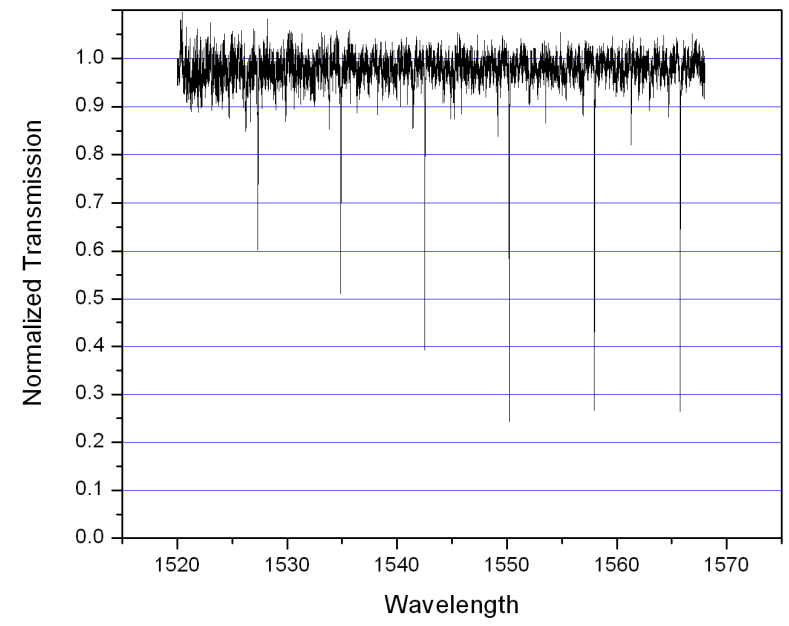

Fig. 2. Transmission spectrum of a replica resonator. Note the easily identifiable free spectral range. 


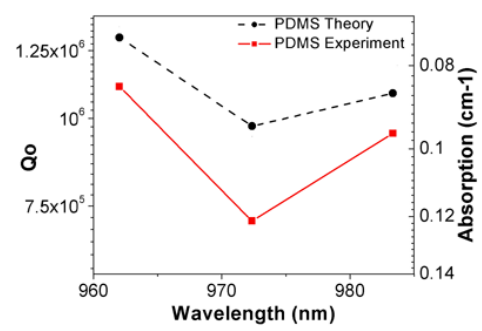

(a)

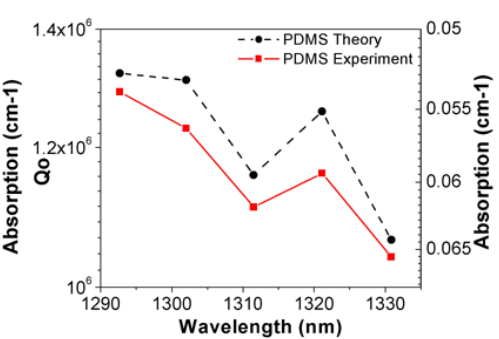

(b)

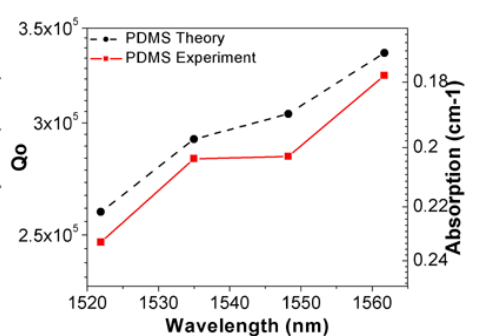

(c)

Fig. 3. Quality factors/Material absorption for PDMS at ) $980 \mathrm{~nm}$, b) $1300 \mathrm{~nm}$ and c) $1550 \mathrm{~nm}$. Q> 1 million achieved at both $980 \mathrm{~nm}$ and $1300 \mathrm{~nm}$.

Therefore, the dominate loss mechanism is the intrinsic material loss, not surface scattering loss. To increase the $\mathrm{Q}$ factor, it is necessary to decrease the material loss. By moving towards the visible, the loss decreases and quality factors above 1 million were achieved with both PDMS and Vicast resonators (figures 3, 4). Using this characterization technique, the loss of Efiron and Crystal Cast were determined at $680 \mathrm{~nm}, 1300 \mathrm{~nm}$ and $1550 \mathrm{~nm}$ (data to be presented).

\section{SUMMARY}

Soft-lithography is able to accurately reproduce both complex 3-D structures and very smooth surfaces, such as lenses and prisms.[9] By combining this technique with an ideal master, such as the ultra-high-Q microtoroid, high-Q microresonators were fabricated. Unlike previous polymer resonators fabricated using molding techniques, $[1,3]$ the quality factor of these microcavities is limited by the material (not by surface scattering), as demonstrated by comparing the measured Q factors with those predicted from material loss data.

Polymer microresonators were fabricated using four different polymers. These polymers were cured using two distinct mechanisms: 1) thermally or 2) UV exposure. Because the PDMS mold is transparent in the UV, the same mold can be used multiple times (interchangeably) for both classes of polymers.

The quality factors and broad band spectra of microresonators were characterized from the visible through the near-IR wavelength range. The highest quality factor achieved was greater than 1 million using a Vicast resonator at $980 \mathrm{~nm}$. The limiting loss mechanism was material absorption.[5, 6]

\section{ACKNOWLEDGEMENTS}

The authors would like to thank Stevens Martin at AOC for the Vicast polymer resin used in this work. This work was supported by DARPA and the Caltech Lee Center. A.M.A is supported by a Clare Boothe Luce postdoctoral fellowship.

\section{REFERENCES}

[1] C. Y. Chao, Guo, L. J., "Polymer microring resonators fabricated by nanoimprint technique," Journal of Vacuum Science Technology B, vol. 20, pp. 2862-2866, 2002.

[2] P. Rabiei and W. H. Steier, "Tunable polymer double micro-ring filters," Ieee Photonics Technology Letters, vol. 15, pp. 1255-1257, 2003.

[3] P. Rabiei, W. H. Steier, C. Zhang, and L. R. Dalton, "Polymer micro-ring filters and modulators," Journal of Lightwave Technology, vol. 20, pp. 1968-1975, 2002.

[4] D. K. Armani, T. J. Kippenberg, S. M. Spillane, and K. J. Vahala, "Ultra-high-Q toroid microcavity on a chip," Nature, vol. 421, pp. 925-928, 2003.

[5] A. L. Martin, D. K. Armani, L. Yang, and K. J. Vahala, "Replicamolded high-Q polymer microresonators," Optics Letters, vol. 29, pp. 533-535, 2004.

[6] A. L. Martin, Shrinivasan, A., Armani, D.K., Min, B., Vahala, K.J., "Micro-molded high-Q polymer resonators for optical loss determination," presented at MRS Symposium Proceedings, San Francisco, CA, 2005.

[7] M. Cai and K. Vahala, "Highly efficient hybrid fiber taper coupled microsphere laser," Optics Letters, vol. 26, pp. 884-886, 2001.

[8] S. M. Spillane, T. J. Kippenberg, O. J. Painter, and K. J. Vahala, "Ideality in a fiber-taper-coupled microresonator system for application to cavity quantum electrodynamics," Physical Review Letters, vol. 91, pp. -, 2003.

[9] Y. N. Xia, E. Kim, X. M. Zhao, J. A. Rogers, M. Prentiss, and G. M. Whitesides, "Complex optical surfaces formed by replica molding against elastomeric masters," Science, vol. 273, pp. 347349, 1996.

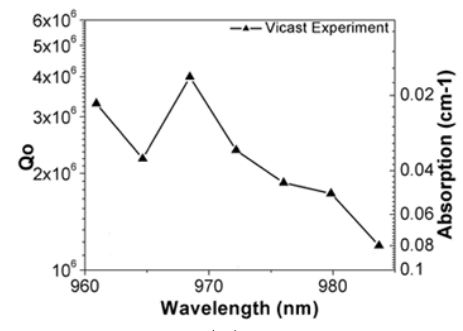

(a)

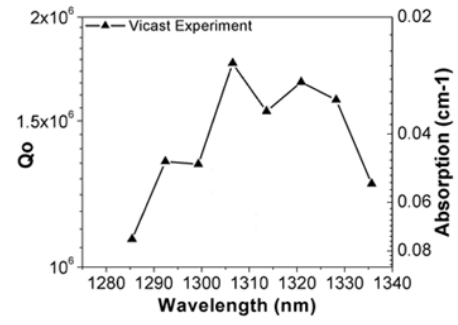

(b)

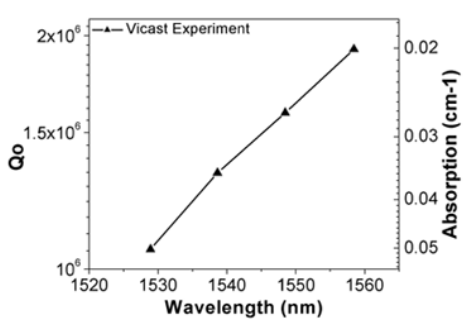

(c)

Fig. 3. Quality factors/Material absorption for Vicast at a) $980 \mathrm{~nm}$, b) $1300 \mathrm{~nm}$ and c) $1550 \mathrm{~nm}$. For Vicast, Q $>1$ million at all wavelengths. 